\title{
New Protocol of Aggressive Packet Combining Scheme
}

\author{
Yaka Bulo \\ Department of Electronics \& \\ Communication Engineering, \\ National Institute of Technology \\ Yupia \\ Arunachal Pradesh
}

\author{
Swarnendu K \\ Chakraborty \\ Department of Computer \\ Science \& Engineering, \\ National Institute of Technology \\ Yupia \\ Arunachal Pradesh
}

\author{
Chandan T Bhunia \\ Department of Computer \\ Science \& Engineering, \\ National Institute of Technology \\ Yupia \\ Arunachal Pradesh
}

\begin{abstract}
Aggressive Packet Combining (APC) scheme is well established for receiving correct packet in high error prone wireless link. In APC three copies of a packet are transmitted and receiver does bit wise majority decision to get the correct copy. The main research challenge in APC is that if two or more copies of the packet become erroneous at the particular bit location then majority logic fails to correct the error. In this paper we propose a new method of correction in APC which will address the limitation which occurs in conventional APC.
\end{abstract}

\section{General Terms}

Error correction capability

\section{Keywords}

Packet combining Scheme, Conventional Aggressive Packet Combining (CAPC) scheme

\section{INTRODUCTION}

In order to combat errors in computer/ data communication networks, ARQ (Automatic Repeat Request) techniques [1-5] with various modifications as applicable to in various communications environments are used. Leung [7] proposed an idea of Aggressive Packet Combining scheme (APC) for error control in wireless networks with the basic objective of fast error control in relatively higher noisy wireless networks. APC is well established and studied elsewhere [3-10]. Several modifications of APC are also reported elsewhere [2-8]. The modifications are due to increasing throughput, tackling various error syndromes and enhancing fast correction. In APC and/ or modified APCs, two or more copies of the packets are transmitted. Copies received by the receiver either error free or erroneous are used in receiver to correct errors by applying Packet Combining schemes differently in different situations. However in original APC, if an error occurs at same locations of erroneous packets, the application of the majority logic as in original APC fails to correct the error. To address the stated problem of APC we propose a new protocol of APC. Analytical results establish that the proposed new scheme provide better correction capability.

\section{REVIEW OF PACKET COMBINING SCHEME (PC)}

Chakraborty [11] suggested a simple technique where the receiver will correct limited error, one or two bit error, from the received erroneous copies. The technique proposed by Chakraborty is illustrated below:

We assume the original transmitted packet as "01010101."

The packet erroneously received by the receiver as "11010101." The receiver requests for retransmission of the received erroneous packet but keeps in store the received erroneous packet. The transmitter retransmits the packet, but again the packet is received by the receiver erroneously as "00010101." Chakraborty proposed that the receiver can correct the error by using two erroneous copies for a bit wise XOR operation between erroneous copies may be performed to locate the error position, in the present example being as follows:

First erroneous copy $\quad 11010101$

Second erroneous copy 00010101

XOR 11000000

The error locations are identified as first and/or second bit from the left. Chakraborty suggested that the receiver can apply brute method to correct error by changing received " 1 " to " 0 " or vice versa on the received copies followed the application of error decoding method in use. In the example the average number of brute application will be 0.5 , and in general $2^{\mathrm{n}-1}$ if $\mathrm{n}$ bits are found in error. Several modifications of PC have been studied elsewhere [12-13] by Bhunia's.

\section{REVIEW OF CONVENTIONAL APC}

Aggressive Packet Combining scheme is a modification of MjPc (Majority Packet Combining) [14]. To illustrate APC it is assumed that an original packet 10101010 is transmitted between a sender and a receiver. In Aggressive Packet Combining (APC) the three copies of packet are sent for each packet between a source and a destination. The Majority logic is applied bit to bit on three copies of the packet. In table: I we have shown different possibilities of APC. In case (1) there is no error in the transmitted three copies. In case (2) receiver receives two copies of correct packet and one copy with an error, so correction is possible by majority logic. In case (3) and case (4) errors are present in two or more copies in which case correction is not possible.

\section{Table:I: Different cases of APC Scheme}

\begin{tabular}{|c|c|c|c|}
\hline Case 1 & Case 2 & Case 3 & Case 4 \\
\hline Copy-1= & Copy-1= & Copy-1= & Copy-1= \\
10101010 & 00101010 & 00101010 & 00101010 \\
Copy-2= & Copy-2= & Copy-2= & Copy-2= \\
10101010 & 10101010 & 00101010 & 00101010 \\
Copy-3= & Copy-3= & Copy-3= & Copy-3= \\
10101010 & 1010110 & 10101010 & 00101010 \\
\hline Correction & Correction & Correction & Correction \\
Probability is & Probability & Probability is & Probability is \\
(1-P3) & is (1-P2)P & (1-P)P2 & P3 \\
\hline Correction & Correction & Correction & Correction \\
not required & possible & not possible & not possible \\
\hline
\end{tabular}




\section{NEW BASIC IDEA}

In this new technique, instead of sending three packets of the original packets as in conventional APC, two packets are sent. If the received two packets are erroneous then XORing of both the erroneous packets are done to locate the error positions. Now receiver sends negative acknowledgement with erroneous bit positions information to the sender and also keeping the copies that has been received erroneously. And instead of retransmitting the whole packets sender sends only the erroneous bit positions by repeating each bit three times so even if the third received packets are erroneous the receiver by using majority logic with three successive bits get the original bits that have been transmitted and erroneous locations are corrected by comparing the bits obtained after majority logic with the erroneous bit locations in the first two received packets. Thus in this technique both horizontal and vertical checking of the received packets are done. This idea will become clearer by the following examples.

Examples:

I.Originalpacket-11001100

$1^{\text {st }}$ erroneous packet-

11011100

$2^{\text {nd }}$ erroneous packet-

10101100

\section{XOR}

01110000

The error locations are identified as $5^{\text {th }}, 6^{\text {th }}$ and $7^{\text {th }}$ bit locations from the right. Now sender sends only the erroneous bits by repeating each bit three times.

The third packet sent is 111000000 and again if it is received erroneously like 101001000 but by majority logic we can get the original string that has been sent like 101-1 $\left(7^{\text {th }}\right)$, 001-0 $\left(6^{\text {th }}\right)$, 000-0 $\left(5^{\text {th }}\right)$. Comparing third packet with the first erroneous packet, the $5^{\text {th }}$ bit in the first erroneous packet is $\mathbf{1}$ but the actual bit is $\mathbf{0}$ so the first corrected packet is 11001100. Now comparing the second erroneous copy with the third packet, the $6^{\text {th }}$ and $7^{\text {th }}$ bits from right of the second packet shown in bold 1 and 0 respectively but the actual bits are 0 and 1 respectively so the $6^{\text {th }}$ and $7^{\text {th }}$ positions are corrected as 0 and 1 from the right. So the corrected packet is 11001100 .

\section{Original packet-10110100}

$1^{\text {st }}$ erroneous packet-

00110100

$2^{\text {nd }}$ erroneous packet10010100

\section{XOR}

10100000

The error locations are identified as the $6^{\text {th }}$ and the $8^{\text {th }}$ locations from the right. After getting negative acknowledgement sender sends the $3^{\text {rd }}$ packet as 111111 and if it is received without error then by majority logic the $6^{\text {th }}$ and $8^{\text {th }}$ bits are 111-1, 111-1 respectively. Comparing third packet with the first erroneous packet, the $8^{\text {th }}$ bit from the right in the first erroneous packet is $\mathbf{0}$ but the actual bit is $\mathbf{1}$ so the first corrected packet is 10110100 .

Now comparing the second erroneous copy with the third packet, the $6^{\text {th }}$ bit from right of the second packet shown in bold is 0 but the actual bit is 1 so the corrected packet is 10110100 .

\section{III.Originalpacket-01101001}

\begin{tabular}{|c|c|}
\hline $1^{\text {st }}$ erroneous packet- & 01001101 \\
\hline $2^{\text {nd }}$ erroneous packet- & 01100101 \\
\hline XOR & 00101000 \\
\hline
\end{tabular}

The error locations are identified as the $4^{\text {th }}$ and the $6^{\text {th }}$ locations from the right. After getting negative acknowledgement sender sends the $3^{\text {rd }}$ packet as 111111 and if it is received with an error then by majority logic the $4^{\text {th }}$ and the $6^{\text {th }}$ bits are 101-1 011-1 respectively. Comparing third packet with the first erroneous packet, the $6^{\text {th }}$ bit from the right in the first erroneous packet is $\mathbf{0}$ but the actual bit is $\mathbf{1}$ so the first corrected packet is 01101001 .

Now comparing the second erroneous copy with the third packet, the $4^{\text {th }}$ bit from right of the second packet shown in bold is 0 but the actual bit is 1 so the corrected packet is 01101001 .

IV.Original packet-11110000

\begin{tabular}{lc}
$1^{\text {st }}$ erroneous packet- & 10110000 \\
$2^{\text {nd }}$ erroneous packet- & $\mathbf{0 1 1 1 0 0 1 0}$ \\
\hline XOR & 11000010
\end{tabular}

The error locations are identified as the $2^{\text {nd }}, 7$ th and the $8^{\text {th }}$ locations from the right. After getting negative acknowledgement sender sends the $3^{\text {rd }}$ packet as 111111000 and if it is received with an error as 111101001 then by majority logic the $2^{\text {nd }}, 7$ th and the $8^{\text {th }}$ bits are $111-1101-1$ 001-0 respectively. Comparing third packet with the first erroneous packet, the $7^{\text {th }}$ bit from the right in the first erroneous packet is $\mathbf{0}$ but the actual bit is $\mathbf{1}$ so the first corrected packet is 11110000 .

Now comparing the second erroneous copy with the third packet, the $2^{\text {nd }}$ and the $8^{\text {th }}$ bits from right of the second packet shown in bold are 1 and 0 respectively but the actual bits are 0 and 1 so the corrected packet is 11110000 .

\section{MATHEMATICAL COMPARISON OF CONVENTIONAL APC AND NEW PROPOSED SCHEME}

In conventional APC original packets are sent three times at a time so in APC $n=3$ and the probability that a packet is in error for conventional APC is given by:

$$
\begin{aligned}
\mathrm{P}_{\mathrm{eC}} & =\left[1-(1-\alpha)^{\mathrm{n}}\right] *\left[1-(1-\alpha)^{\mathrm{n}}\right] *\left[1-(1-\alpha)^{\mathrm{n}}\right] \\
\mathrm{P}_{\mathrm{eC}} & =\left[1-(1-\alpha)^{\mathrm{n}}\right]^{3}
\end{aligned}
$$

Where $\alpha$ is bit error rate (BER) and $\mathrm{N}$ is the number of bits in a packet.

In our proposed scheme instead of sending three packets, two packets are sent and if the packets are received erroneous then by XORing operation erroneous bit positions are found. And in the $3^{\text {rd }}$ packet sender sends the bits which are in error by repeating each bit three times but not the whole packet. At the receiver side receiver performs majority logic of the received packets and starts horizontal checking starting from LSB bit. 
In this scheme the probability that a packet is in error $\left(\mathrm{P}_{\mathrm{eN}}\right)$ is given by

$\mathrm{P}_{\mathrm{eN}}=\left[1-(1-\alpha)^{\mathrm{n}}\right]^{2} *\left[1-\left(1-\alpha^{3}\right)^{\mathrm{n}}\right]$

By using this new technique correction capability of APC increases more than that of the conventional APC.

\section{SIMULATION RESULTS}

Curves (Fig. 1 to 4 ) have been plotted for probability of packets in error for various values of $n$ for conventional APC $\left(\mathrm{P}_{\mathrm{eC}}\right)$ and new proposed scheme $\left(\mathrm{P}_{\mathrm{eN}}\right)$ and various values of bit error rate $(\alpha)$ from $10^{-4}$ to $10^{-1}$. We measure the performance in terms of error correction capability.

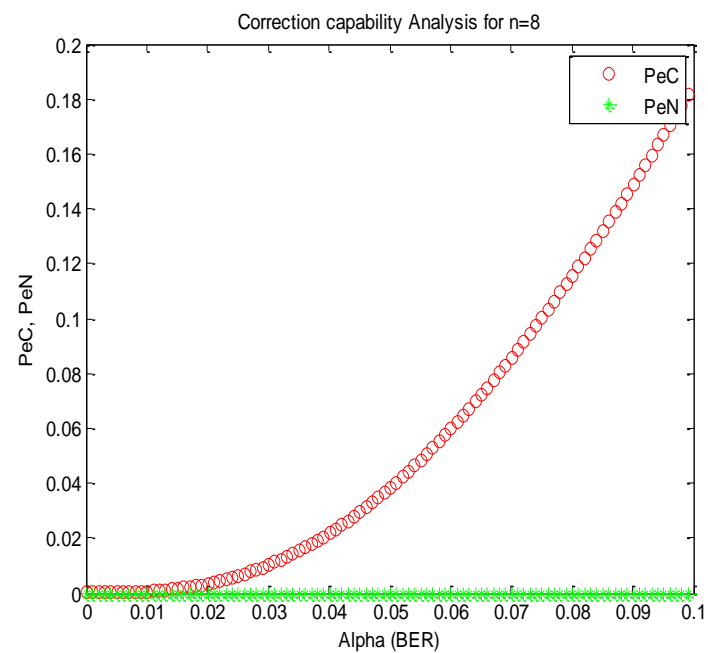

Fig.1 for $n=8$

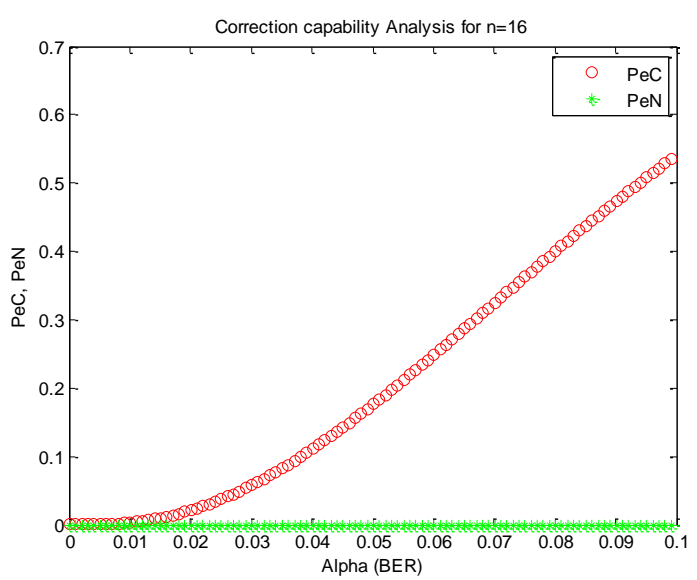

Fig. 2 for $n=16$

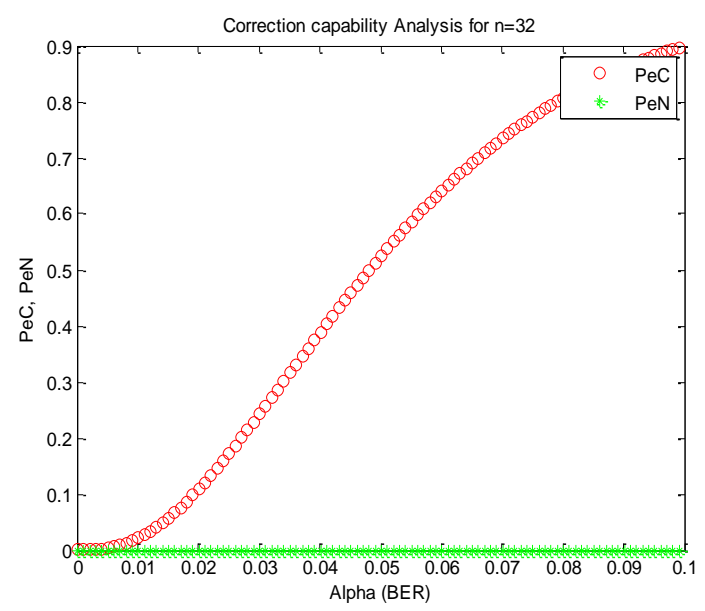

Fig. 3 for $n=32$

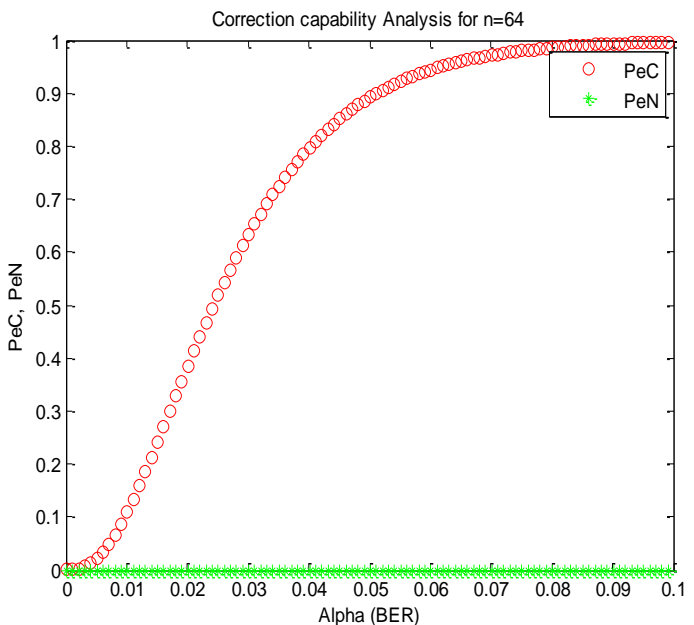

Fig. 4 for $n=64$

\section{CONCLUSIONS}

In this paper, a new scheme of APC is proposed and studied. The new proposed scheme provides better correction capability than the conventional APC as observed from the results. Correction capability of this proposed scheme increases: (a) with increase in $n$ (number of bits in packet), (b) with increase in bit error rate $(\alpha)$ as observed from simulation results.

Thus the performance of this scheme is superior than the conventional APC.

\section{REFERENCES}

[1] C T Bhunia, A Few Modified ARQ Techniques, Proceedings of the International Conference on Communications, Computers \& Devices, ICCCD-2000, 14-16, December' 2000, IIT, Kharagpur, India, Vol.II,pp.705-708.

[2] C T Bhunia, ARQ Techniques: Review and Modifications, Journal IETE Technical Review, SeptOct' 2001 vol 18,No 5,pp 381-401

[3] R J Beniece and A H Frey Jr, Analysis of retransmission schemes, IEEE Trans CommTech, COM-12, pp 135-145, Dec 1964. 
[4] S Lin, D Costello Jr and M J Miller, Automatic repeat request error control schemes, IEEE Comm Mag, 22, pp 5-17, Dec'1984.

[5] A R K Sastry, Improving Automatic Repeat Request performance on satellite channels under high error rate conditions, IEEE Trans Comm, April' 77, pp 1-10.

[6] C T Bhunia Modified Aggressive Packet Combining Scheme, Pre-print, ICTP, Italy, IC/2010/037, pp1-10.

[7] Yiu-Wing Leung, Aggressive Packet Combining for error control in wireless networks, trans. Comm vol. E83, No 2 Feb' 2000, pp38-385.

[8] Don Towsly, The shutter Go Back-N ARQ Protocol, IEEE Trans Comm, Vol 27, No 6, June' 79, pp 869-875.

[9] N D Birrell, Pre-emptive retransmission for communication over noisy channels, IEEE Proc Part F, Vol 128, 1981, pp 393-400.

[10] H Brunel and M Maeneclacey, On the throughput performance of some of continous ARQ strategies with repeated transmissions, IEEE Trans Comm, Vol Comm 34, 1986, pp 244-249.

[11] Shyam S. Chakraborty et al, An ARQ Scheme with Packet Combining, IEEE Comm Letters, Vol 2, No 7, July'95,pp 200-202.

[12] C T Bhunia Modified Aggressive Packet Combining Scheme using Error Forecasting Decoding to combat error in network. Pro. ICITA' 05(Proc. IEEE Computer soc.), Sydney, Vol 2, 4-7, July 2005, pp641-646.

[13] C T Bhunia, Packet Reversed Packet Combining Scheme , Proc. IEEE Computer soc., CIT' 07, Aizu University, Japan, pp 447-451

[14] SB Wicker, Adaptive rate error control through the use of diverse combining and majority logic decoding in hybrid ARQ Protocol, IEEE Trans Comm, Vol.39, No 3, March 1991, pp 380-385 